\title{
CURRENT STUDIES AND FUTURE PROSPECTS IN STELLAR-STRUCTURE IMAGING WITH THE GI2T
}

\author{
PH. STEE, D. BONNEAU, F. MORAND, D. MOURARD \\ AND \\ F. VAKILI \\ Equipe GI2T \\ Observatoire de la Côte d'Azur \\ 2130 route de l'Observatoire, Caussols, \\ 06460 St Vallier de Thiey, France. \\ email: stee@rossini.obs-nice.fr
}

\begin{abstract}
The capability of optical long baseline interferometry for measuring the angular diameter of stars or binary separation is now well established. However, for the imaging of photospheric disk structures one needs very long baseline interferometers operated in the multi-telescope phaseclosure technique. In this paper we will stress the capability of spectrointerferometric measurements to constrain the physics of hot stars. We will report our study of the interacting binary, $\beta$ Lyrae, and the masslosing Be star $\gamma$ Cassiopeiae. We will look at the interpretation of both the modulus and phase data recorded by the long baseline interferometer GI2T in the southern France. The performances and limitations of spectrointerferometric techniques will also be discussed through some of the most exciting prospects within the reach of current interferometers.
\end{abstract}

\section{The $\beta$ Lyrae campaign}

$\beta$ Lyrae is a puzzling Be and eclipsing binary of 12.9 days orbital period on which a huge amount of spectroscopic and photometric observations has been accumulated for more than 100 years. A realistic picture of this complex system is now being proposed with a set of basic physical parameters determined (Harmanec \& Scholz, 1993) and models proposed to explain 
TABLE 1. $\beta$ Lyrae 1994 international campaign

\begin{tabular}{ll}
\hline Interferometry & GI2T, Station de Calern, OCA, France \\
\hline Spectroscopy & $2.00 \mathrm{~m}$, Ondrejov, Czech Republic \\
& $1.22 \mathrm{~m}$, UBC-DAO, Canada \\
\hline Spectro-polarimetry & $1.83 \mathrm{~m}$, UBS-DAO, Canada \\
\hline Photometry & $0.65 \mathrm{~m}$, Hvar, Croatia \\
& $0.40 \mathrm{~m}$, Vanderbilt, Mt Hopkins, USA \\
& $0.25 \mathrm{~m}$, Villanova, Mt Hopkins, USA \\
& $0.76 \mathrm{~m}$, Jungfraujoch, Switzerland \\
\hline
\end{tabular}

the eclipsing geometry and the complex spectral behavior of this object (Mazzali et al., 1992; Hubeny \& Plavec, 1991).

$\beta$ Lyrae is presently described as a close binary with strong interaction between the components. Now, it is generally admitted that the primary is a Be star surrounded by an accretion disk fead by the matter lossed by the secondary, a bright giant B filling its Roche lobe.

This stellar system offers a unique opportunity to study the processes involving mass exchange related to evolution of a close binary. However, at this time, some points remain unclear, for instance:

1. The origin of the optical and UV emission lines,

2. The nature of the source for the radio and $\mathrm{X}$ ray emissions,

3 . The mechanism producing the long term variations,

4. The structure and the physical state of the components and the circumstellar mater.

To progress in the knowledge of $\beta$ Lyrae, an international campaign has been organized by Petr Harmanec of the Ondrejov Observatory during the 1994 observing season.

Interferometric, spectroscopic, photometric and polarimetric observations have been carried out by differents groups in Europe and North America (Table 1). The first results of the analysis of this observations are presented by Harmanec et al. (1995).

Interferometric observations of $\beta$ Lyrae have been done with the GI2T operated at the Calern observatory (Mourard et al. 1994a). The GI2T is a Michelson stellar interferometer with 2 large $(\mathrm{D}=1.5 \mathrm{~m})$ apertures on a North-South variable baseline, combining a high angular resolution (close to $1 \mathrm{mas}$ ) and a medium spectral resolution $(\mathrm{R}=4500)$ (Mourard et al. 1994a). The initial goals of this were targetting to resolved the binary system in the 


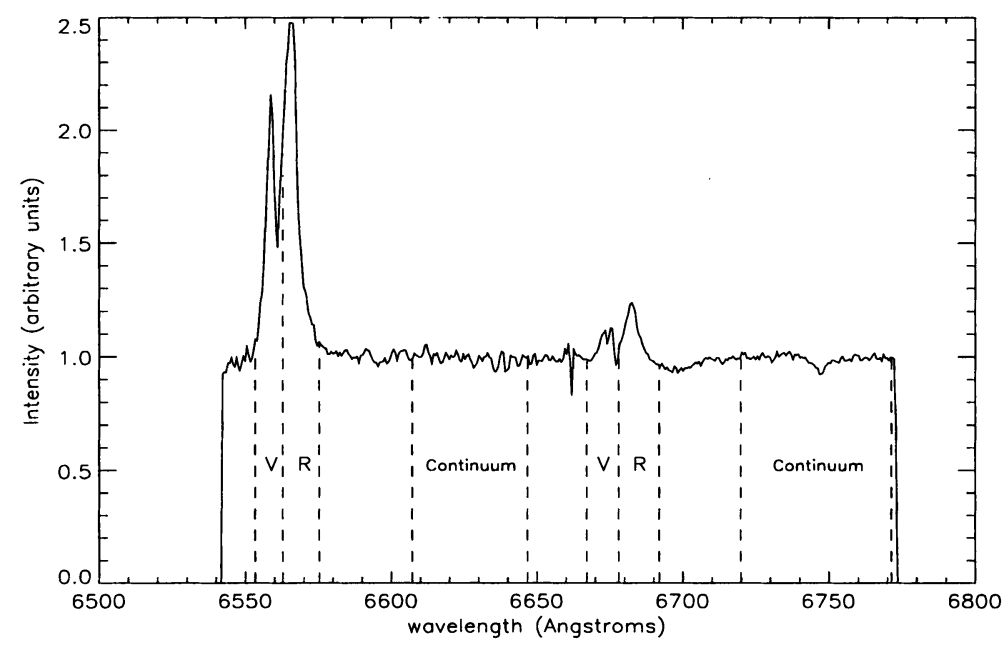

Figure 1. GI2T spectrum of $\beta$ Lyr obtained the 1994, July, 17 showing the spectral bandpass selected for the interferometric analysis.

continuum and to characterize the morphology of the regions which emit the strong $\mathrm{H} \alpha$ and $\mathrm{He}$ I $6678 \AA$ lines. The interference fringes recorded in dispersed speckles are analysed (Mourard et al. 1994b) to compute the observed visibilities of the source in narrow spectral bands taken in the lines (whole line, violet and red peaks) and in the continuum (Figure 1 and 2).

To search for spectral or time variations of the angular extension of the source, the observed visibility have been calibrated to obtain an estimate of the modulus of the visibilty function of the source, according to:

$$
V_{\text {cal }}(\beta L y r a e)=V_{\text {obs }}(\beta \text { Lyrae }) \frac{V_{\text {ref }}(\gamma \text { Lyrae })}{V_{\text {obs }}(\gamma \text { Lyrae })} .
$$

In order to apply Eq.(1), we have systematically observed the reference star $\gamma$ Lyrae before and after $\beta$ Lyrae.The photometric determination of the angular diameter of $\gamma$ Lyrae, $\phi_{\text {ref }}=0.7$ mas (Legett et al., 1986), assumed to be constant, provides the value $V_{\text {ref }}=0.92$ at $\lambda=6600 \AA$ for the 51 meters baseline, used during the observations.

From the analysis of the interferometric data, one concludes that: 
194 PH. STEE, D. BONNEAU, F. MORAND, D. MOURARD AND F. VAKILI
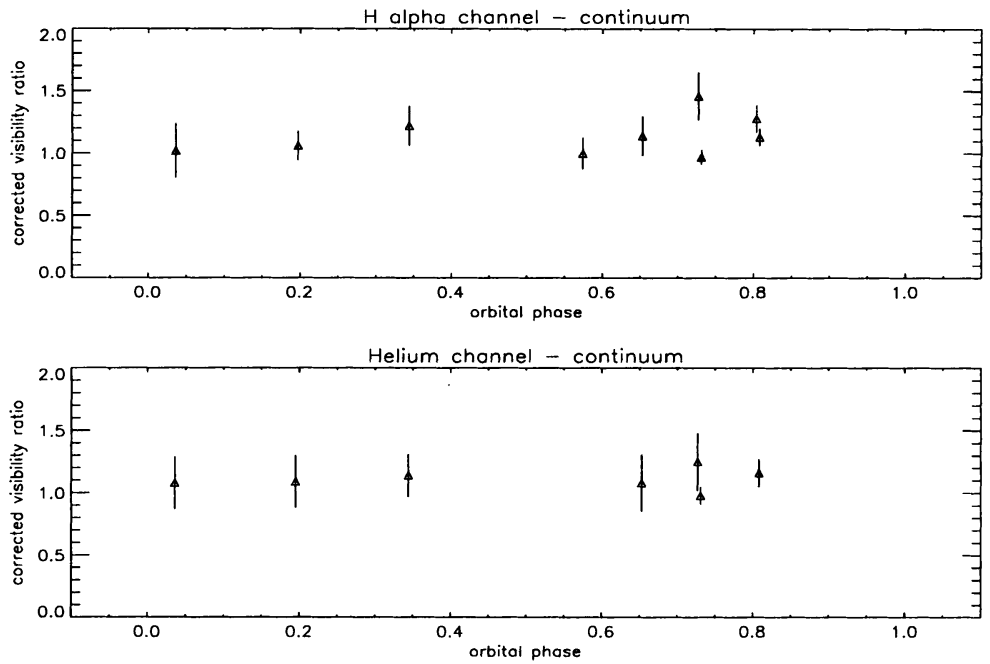

Figure 2. GI2T calibrated visibilities of $\beta$ Lyr in the H $\alpha$ (top) and He I $6678 \AA$ lines (bottom) as a function of the orbital phase.

1. In the continuum, the calibrated visibility is close to 1.0 at all orbital phases, meaning that the source is unresolved in the N-S direction (size $\ll 0.7 \mathrm{mas}$ ). This results agrees with the spatial orientation of the orbital axis $\theta \sim 160^{\circ}$ close to the N-S direction deduced from polarimetric observations (Rudy, 1979).

2. In the emission lines, the calibrated visibility is found to be less than 1.0 at all observed orbital phases. For the whole lines, we found $V_{\text {cal }} \sim$ 0.55 at $\mathrm{H} \alpha$ and $V_{\text {cal }} \sim 0.75$ at HeI $6678 \AA$ the source beeing resolved in the $\mathrm{N}-\mathrm{S}$ direction. Moreover, the visibility in $\mathrm{V}$ and $\mathrm{R}$ peaks appears to be smaller than the visibility in the whole lines. This result indicates that the lines could be formed, at least partly, in regions elsewhere than the source of the continuum, probably located outside the orbital plane.

These preliminary results have clearly shown the importance of combining angular and spectroscopic observations at high resolution for studying a star with a complex morphology as a close and interacting binary. However, the initial goals of the interferometric observations of $\beta$ Lyrae have not yet been reached. New observations should be carried out to resolve the components of the binary and detail the morphology of the sources emitting $\mathrm{H} \alpha$ and HeI lines. First, by improving the data analysis to measure the wavelength-dependent variation of the phase of the complex visibility func- 
tion across the lines. Indeed, at the first order, this phase informs us about the position of the emitting regions relative to the source of the spectral continuum. Second, by improving the instrument, in order to:

1. Allow a two dimensional spatial frequency coverage (increasing the duration of the observation to benefit of the super-synthesis effect or by adding an East-West baseline).

2. Increase the angular resolution by the use of a longer baseline.

\section{Active early type stars}

We will now illustrate the way in which long baseline interferometry can be used to constrain a radiative wind model that we have developed at the Observatoire de la Côte d'Azur and adapted to the active early type (Be) star $\gamma$ Cassiopeiae.

\subsection{WHAT IS A BE STAR?}

Be stars are non-supergiant B-type stars whose spectrum has, or had at some time, one ore more Balmer lines in emission (Collins, 1986). They are presumed to be fast rotators spinning at $\sim 200 \mathrm{kms}^{-1}$ or more (i.e. between 0.5 to 0.9 of their critical velocity) and have a large stellar wind and high rate of mass loss.

This stellar wind is at the origin of a two components envelope, which is characterized by an equatorial plane with high density and low expansion where the Balmer emission lines are supposed to be formed. Whereas, a polar region with low density and high expansion is supposed to be responsible for UV absorption line profiles that are Doppler shifted with velocities up to $2000 \mathrm{kms}^{-1}$. This envelope also produce a linear polarization by free electrons of about $\sim 1.0-1.5 \%$, and an IR excess that has been measured by IRAS.

Numerous ad-hoc models attempting to reproduce isolated characteristics of Be stars have been proposed in the past (Poeckert and Marlborough 1978; Waters \& Marlborough 1992). They try to fit a few observable features by constraining as many free parameters as possible. We propose a strategy which lies between a totally ad-hoc and a physically consistent approach which starts from the hydrodynamic equations in the polar regions in order to provide the density distribution and the velocity fields throughout the envelope. Moreover, this approach enables us to produce intensity maps as a function of wavelength and introduce high angular resolution data in our modelling by computing theoretical visibilities. 


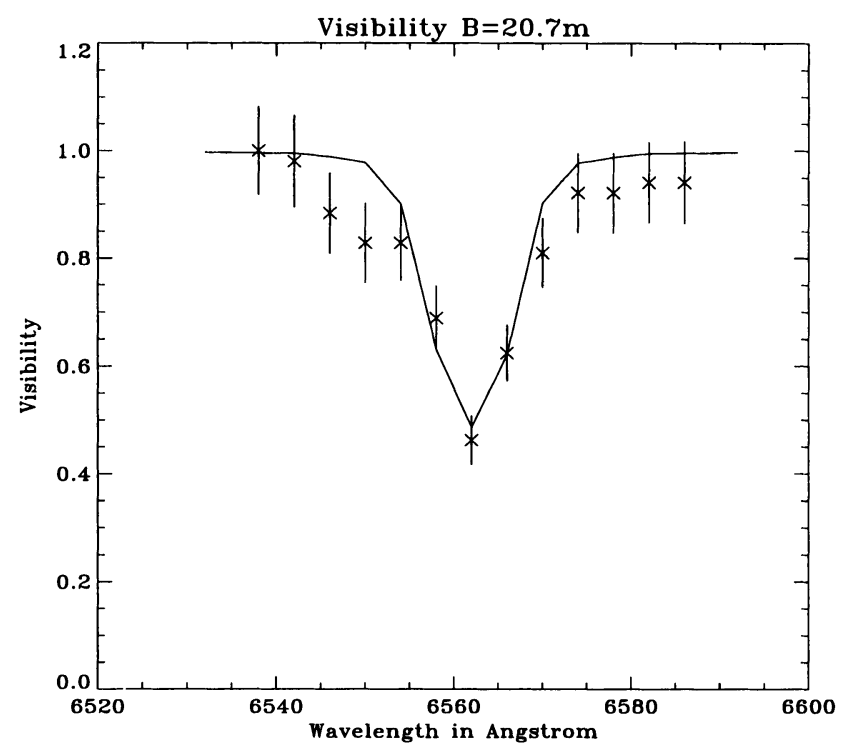

Figure 3. Visibilities corresponding to the Doppler-shifted maps across the $\mathrm{H} \alpha$ line profile for the 20.7 meter baseline. Solid line: model, crosses: actual data from the GI2T.

\subsection{A LATITUDE DEPENDENT RADIATIVE WIND FOR $\gamma$ CAS}

We have built a model producing both spectroscopic and interferometric synthetic data that have been compared to the GI2T observations. This model is a latitude-dependent radiative wind numerical code for Be stars which clearly shows that the morphology of the circumstellar envelope depends strongly on the central observational wavelength and bandwidth (Araújo \& Freitas Pacheco 1989; Araújo et al. 1994; Stee \& Araújo 1994). The line force in our model is the same as used by Friend \& Abbott (1986), but we introduce a varying contribution of thin and thick lines from pole to equator by adopting radiative parameters which are latitude-dependent. The velocity fields and density relationships derived from the hydrodynamic equations are then used for solving the statistical equilibrium equations. By adopting the Sobolev approximation, we could easily obtain a good estimate of both electronic density and hydrogen level populations throughout the envelope. The numerical calculation was performed for parameters characterisic of the Be star $\gamma$ Cassiopeiae.

We have obtained an $\mathrm{H} \alpha$ emission profile from our rotating and expanding wind model that is in good agreement with the observed spectra. Our computed intensity maps, in both the continuum and $\mathrm{H} \alpha$, agree reasonably well with maps derived from actually observed visibilities. We have 
also computed intensity maps across the $\mathrm{H} \alpha$ line in order to obtain the change in visibility as a function of Doppler shift across the emission line. The maps are symmetrical around the central wavelength due to the fact that our model is axi-symmetric. A comparison with the visibility obtained from these maps and the GI2T data is shown in Fig. 3 for the 20.7 meter baseline. The agreement is good within errors bars of the data. This agreement is important because due to the structure of the velocity field in the envelope, its morphology strongly depend on the central observational wavelength and bandwidth (see also Stee \& Araújo 1994). Finally the model indicates that a radiative wind, driven mainly by optically thin lines at the equator, is a likely scenario for $\gamma$ Cas. This is discussed in greater depth in Stee et al. (1995).

\subsection{CONNECTION BETWEEN THE STELLAR PHOTOSPHERE AND THE CIRCUMSTELLAR ENVIRONMENT}

One limitation of our approach is the stationary hypothesis introduced in our model. Be stars exhibit well-known temporal variations at time scales and amplitudes ranging from minutes to decades and a few percents to several ten percents respectively. The origin of these variations is not well understood at the present time. Short term variations are often considered to relate to transient photospheric activities. The envelope is fueled in material by some energetical mechanism like the magnetic field which enhance the mass-loss and in return should be echoed by the physical conditions in the envelope. Photometric or spectroscopic semi-regular variations due to rotation or Non-Radial Pulsations (NRP) should be clocked by the typical period of these phenomena and could ultimately become detectable by means of spectro-interferometry. For instance, the direct detection of high order NRP's modes could in principle become feasible with a very long baseline optical interferometer. A few words can be said here: NRP's must modulate at some level the emerging flux of the photospheric disk. The spatial filter which is the interferometer could be matched to the spatial scale of the pulsationnal mode and a single observation would detect the hypothetical presence of the NRP which modulates the photospheric disk. At the other extreme, long term variations of the $V / R$ Balmer, Helium or Fe lines are generally attributed to one-armed density waves propagating in nearly Keplerian disk (Okasaki 1991) and are opposed to pulsating envelopes (Doazan 1987). Spectrally resolved interferometry can adress this question by following directly the variations of the envelope size and correlating it with the $\mathrm{V} / \mathrm{R}$ parameter. 


\section{Conclusion and prospects for photospheric structures imaging}

There is a strong scientific case for observing stellar objects with very long optical interferometers operated on the ground and in space. Today the presence of stellar structures other than our Sun is inferred from photometric or line profile variability at various wavelengths. Inversion Doppler and Zeeman imaging techniques apply nowadays to a variety of stellar objects like Ap-Bp stars, RS CVn's, T Tau or Herbig PMS objects. These techniques are somehow limited by the inclination of the rotational axis with respect to the line of sight, etc... A stellar interferometer with even a limited number of apertures and directions can dramatically improve these limitations bringing unique insight to their understanding.

\section{References}

Araújo F.X., Freitas Pacheco J.A., 1989, MNRAS 241, 543.

Araújo F.X., Freitas Pacheco J.A., Petrini D., 1994, MNRAS 267, 501

De Greve J.P., Linnell A.P., 1994, A\&A 291, 786

Doazan V., 1987, IAU Colloquium 92, ed. A. Sletteback and T.P. Snow, Cambridge, Cambridge University Press, p 279

Friend D.B., Abbott D.C., 1986, ApJ 311, 701

Harmanec P., Scholz G., 1993, A\&A 279, 131

Harmanec et al., 1995, submitted to A\&A

Hubeny I., Plavec M.J., 1991, AJ 102, 1156

Hummel C.A., Armstrong J.T., Quirrenbach A., Buscher D.F., Mozurkewich D., Elias II N.M., Wilson R.E., 1994, AJ 107, 1859.

Legett S. K., Mountain C.M., Selby M.J., Blackwell D.E., Booth A.J., Haddock D.J., Petford A.D., 1986, A\&A 159, 217

Mazzali P. A., Pauldrach A.W.A., Puls J., Plavec M.J., 1992, A\&A 254, 241

Mourard D., Tallon-bosc I., Blazit A., Bonneau D., Merlin G., Morand F., Vakili F., Labeyrie A., 1994a, A\&A 283, 705

Mourard D., Tallon-bosc I., Rigal F., Vakili F., Bonneau D., Morand F., Stee Ph., 1994b , A\&A 288, 675

Okazaki T., 1991, PASJ 43, 75

Rudy J. R., 1979 MNRAS 186, 473

Schwarzchild M., 1974, ApJ 195, 137.

Stee Ph., Araújo F.X., 1994, A\&A 292, 221.

Stee Ph., Araújo F.X., Vakili F., Mourard D., Arnold L., Bonneau D., Morand F., TallonBosc I., 1995, A\&A 301, 219.

Vakili F., Mourard D., Stee Ph., 1994, IAU Symp. 162, Pulsation, Rotation and Mass Loss in Early Type Stars, (Kluwer: Holland), pp. 435 